\title{
A Wavelet Based Approach to Solar-Terrestrial Coupling
}

\author{
Ch. Katsavrias ${ }^{1}$, A. Hillaris ${ }^{1}$, P. Preka-Papadema ${ }^{1}$ \\ ${ }^{1}$ Department of Astrophysics, Astronomy and Mechanics, Faculty of Physics, University \\ of Athens, Panepistimiopolis Zografos (Athens) , GR-15783, Greece
}

\begin{abstract}
Transient and recurrent solar activity drive geomagnetic disturbances; these are quantified (amongst others) by $\mathrm{D}_{\mathrm{ST}}$, AE indices time-series. Transient disturbances are related to the Interplanetary Coronal Mass Ejections (ICMEs) while recurrent disturbances are related to corotating interaction regions (CIR). We study the relationship of the geomagnetic disturbances to the solar wind drivers within solar Cycle 23 where the drivers are represented by ICMEs and CIRs occurrence rate and compared to the $\mathrm{D}_{\mathrm{ST}}$ and $\mathrm{AE}$ as follows: terms with common periodicity in both the geomagnetic disturbances and the solar drivers are, firstly, detected using continuous wavelet transform (CWT). Then, common power and phase coherence of these periodic terms are calculated from the cross-wavelet spectra (XWT) and waveletcoherence (WTC) respectively. In time-scales of $\approx 27$ days our results indicate an anti-correlation of the effects of ICMEs and CIRs on the geomagnetic disturbances. The former modulates the $\mathrm{D}_{\mathrm{ST}}$ and $\mathrm{AE}$ time series during the cycle maximum the latter during periods of reduced solar activity. The phase relationship of these modulation is highly non-linear. Only the annual frequency component of the ICMEs is phase-locked with $\mathrm{D}_{\mathrm{ST}}$ and AE. In time-scales of $\approx 1.3-1.7$ years the CIR seem to be the dominant driver for both geomagnetic indices throughout the whole solar cycle 23.
\end{abstract}

Keywords: Magnetosphere, Geomagnetic Disturbances, Solar Cycle, Solar Wind, Wavelet Coherence

\footnotetext{
${ }^{*}$ Corresponding author

Email address: ckatsavrias@phys.uoa.gr (Ch. Katsavrias $\left.{ }^{1}\right)$

${ }^{1}$ Tel. 00302107276855
} 


\section{Introduction}

The connection of solar activity to geomagnetic disturbances, dubbed Solar-Terrestrial Coupling, remains an open field of research. The effects on Earth appear as geomagnetic disturbances driven by the solar windmagnetosphere interaction and quantified by geomagnetic indices (see review by Akasofu, 2011).

Feynman (1982) and $\mathrm{Du}(2011)$, indicated that the annual values of the geomagnetic index $a a$ could be the resultant of two components: one originating from solar transient (or sporadic) activity and in phase with the solar cycle; the other was related to recurrent solar drivers with peak in the declining phase (see also Richardson and Cane, 2012). Along the same line Cliver (1995) provides a historical review of the solar-terrestrial research since 1930, and the two basic types of geomagnetic storms: recurrent and sporadic. The studies, mentioned above, propose two classes of geomagneticsolar drivers on a time scale of approximately a year as Feynman (1982) and $\mathrm{Du}$ (2011) used annual averages of $a a$ in their study. The interplanetary coronal mass ejection (ICME) is the major driver of transient geomagnetic activity. The solar recurrent activity, on the other hand, is driven by High Speed Solar Wind Streams (HSSWS) and Co-rotating Interaction Regions (CIR) (Schwenn, 2006; Pulkkinen, 2007). Borovsky and Denton (2006) and Richardson and Cane (2012) indicate, also, that the different driver classes (CIR, ICME) result in distinct geomagnetic disturbances; the ICMEs, for example, induce higher ring current, manifested by a high negative peak in $\mathrm{D}_{\mathrm{ST}}$.

The solar-geomagnetic coupling, when studied in the frequency plane manifests itself with periodic terms having the same periodicity in the solar drivers and the geomagnetic indices time series. The basic periodicity is the $11 / 22$ year solar cycle (sunspot and magnetic respectively), yet quasi-periodic variations on shorter time-scales have been reported.

Lou et al. (2003) found $\mathrm{A}_{p}$ index periodicities of 187, 273 and 364 days in the 1999-2003 time interval. Periodicities of about 27.5, 13.5, 9.1, and 6.8 days, due to the solar rotation have been identified in the solar wind speed and the IMF polarity (Gonzalez and Gonzalez, 1987; Clúa de Gonzalez et al., 1993; Svalgaard and Wilcox, 1975; Fenimore et al., 1978; Sabbah and Kudela, 2011). Kudela et al. (2010) reported that a range of periodicities, 
1.7-2.2 years, appear in cosmic rays during the time interval 1951-2010, while Mavromichalaki et al. (2003) published similar results for the 1953-1996 interval. Valdés-Galicia et al. (1996); Mursula (1999) and Prabhakaran Nayar et al. (2002) reported different periodic variations of the geomagnetic activity index $\mathrm{A}_{p} ; 1.3-1.4$ years during even cycles and of 1.5-1.7 years during odd ones.

Katsavrias et al. (2012) examined the 1966-2010 time period for periodicity in the solar activity, the solar wind speed, interplanetary magnetic field and the geomagnetic indices using wavelet analysis. Within the examined timeseries time-localized common spectral peaks, between the fluctuations in the solar wind characteristics and the geomagnetic indices were detected. Certain periodicities were dominant within specified intervals which, at times, were different for different geomagnetic indices.

The interdependence between different time series requires a different wavelet based approach. In this case cross wavelet transform and wavelet coherence (XWT and WTC Grinsted et al. 2004) are used for the quantification of the interdependence. This approach has been, already, used in the study of common periodicities between two time-series and the corresponding phase relationship between them. Valdés-Galicia and Velasco (2008) studied the coherence of the sunspots with open solar magnetic fluxes. Deng et al. (2012) investigated the coronal index-sunspot numbers phase relationship finding coherent behaviour in low-frequency components corresponding to the 11-year Schwabe cycle; this coherence was absent in the high-frequency components. Deng et al. (2013) applied this method between $10.7 \mathrm{~cm}$ solar radio flux and sunspot numbers from 1947 February to 2012 June; the phase relationship between the time series was found both time and frequency dependent.

In this work a refinement of the Katsavrias et al. (2012) wavelet based approach is presented which aims at the detection of common and coherent periodicity and phase relationship between the ICMEs, CIRs and the $\mathrm{D}_{\mathrm{ST}}$, AE geomagnetic indices time-series by means of cross wavelet transform and wavelet coherence calculations.

\section{Data Selection}

We used time-series of the occurrence rate of the geomagnetic drivers, ICMEs, CIR and of different geomagnetic indices, representative of the conditions in the magnetosphere, as follows: 

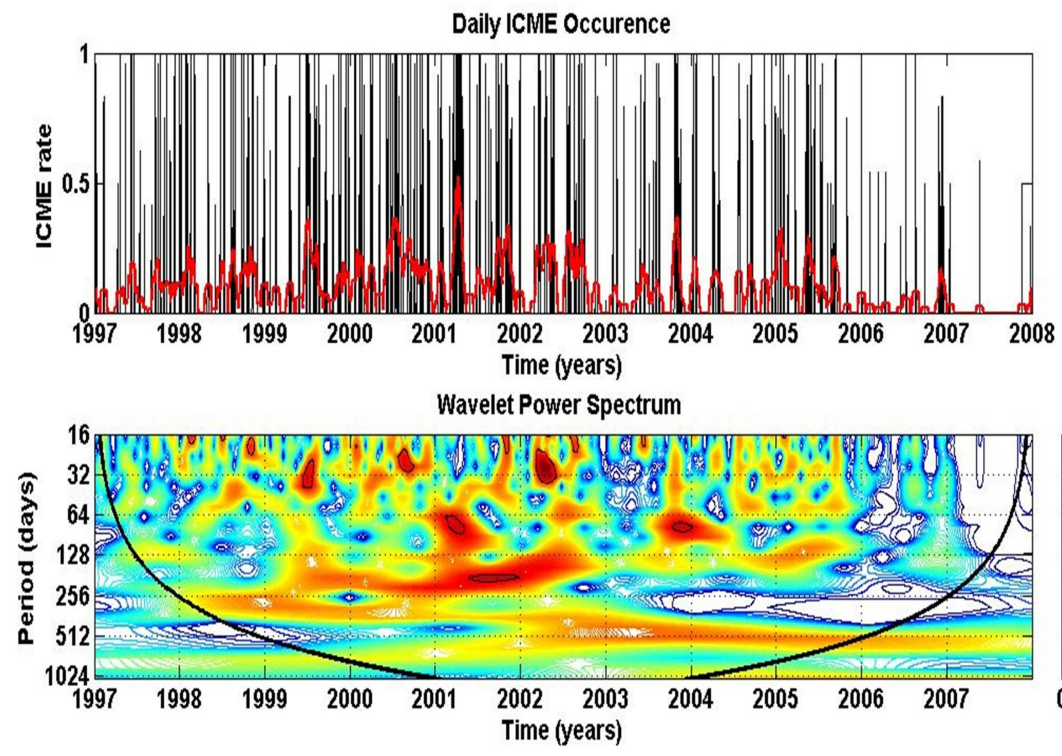

Global Wavelet Spectrum

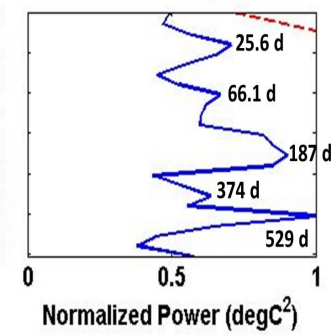

Figure 1: Time-series (upper panel), Wavelet power (lower panel, left) and global wavelet spectra (lower panel, right) of ICMEs occurrence; the red line is the 27-days moving average smoothed time-series. The Wavelet power display is colour-coded with red corresponding to the maxima; the black contour is the cone of influence of the spectra, where edge effects in the processing become important. The dashed line in the global spectra represent a confidence level above $95 \%$. 


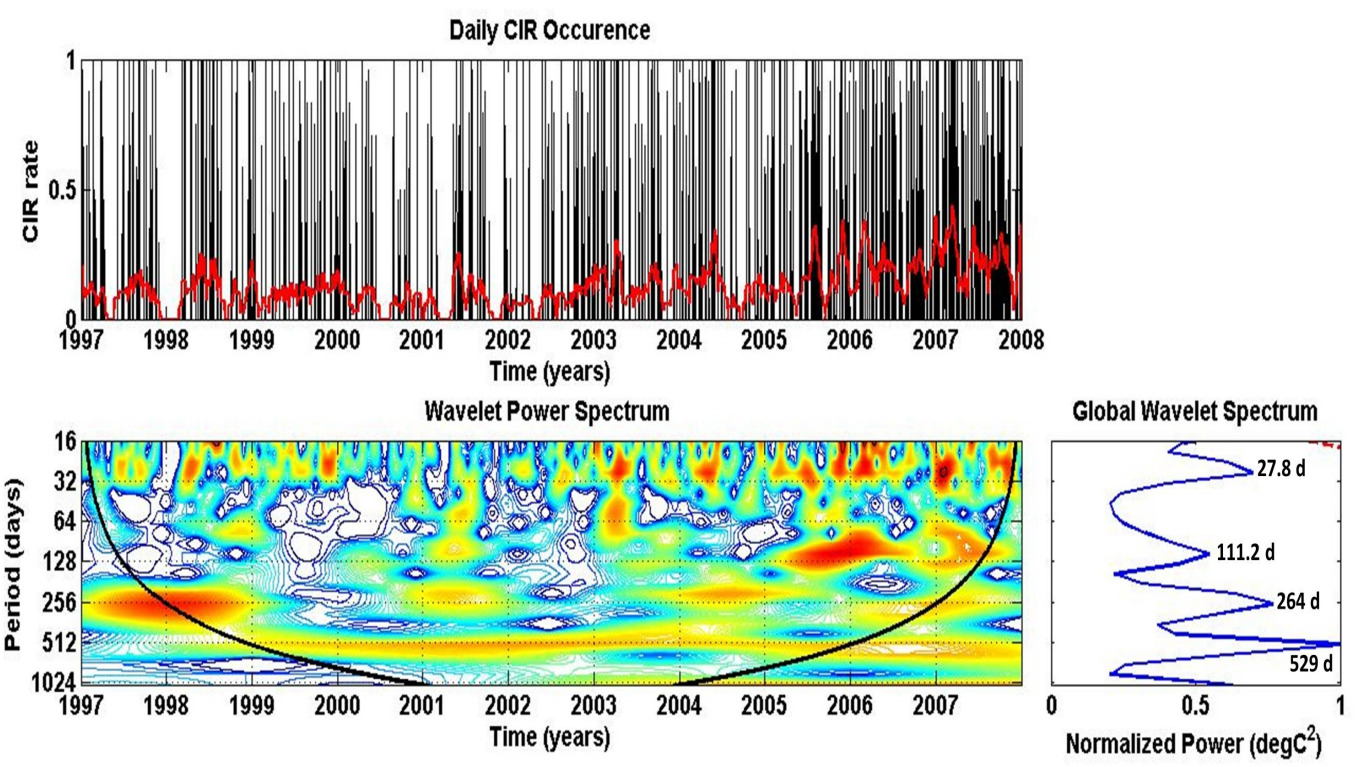

Figure 2: Same with figure 1 but for CIR occurrence.

- ICMEs per day from the Jian et al. (2006a) catalogue on line ${ }^{2}$. The daily rate is the duration of the ICME passage on that day, in hours, divided by 24. Two more ICME lists by Richardson and Cane (2010) and Mitsakou and Moussas (2014) were available yet the selection does not affect our analysis as the three lists differ little from each other and exhibit the same trends in the ICME occurrence rate (Mitsakou and Moussas, 2014).

- CIRs per day from the Jian et al. (2006b) list on lin£? The daily rate is the duration of the CIR passage on that day, in hours, divided by 24 defined similarly to the ICME rate in the previous bullet. We selected CIRs because their geomagnetic effectiveness is greater, on average, than the other stream interaction regions.

- Geomagnetic indices from the OMNIweb database: The $\mathrm{D}_{\mathrm{ST}}$, represents the strength of the Earth ring current; values below -30 nT indi-

\footnotetext{
${ }^{2} \mathrm{http}: / /$ www-ssc.igpp.ucla.edu/jilan/STEREO/Level3/STEREO_Level3_ICME.pdf recent updates of the catalogue extend beyond 2006.

${ }^{3}$ www-ssc.igpp.ucla.edu/jilan/STEREO/Level3/STEREO_Level3_SIR.xls
} 
cate a geomagnetic storm. The AE quantifies sub-storms as it represents auroral electrojet intensity (Mayaud, 1980).

Our data-set covers solar cycle 23, from January 1st, 1997 to December 31st, 2007, and consists of daily average values.

\section{Results and Discussion}

\subsection{Wavelet analysis}

The analysis of a function in time, be it $\mathrm{F}(\mathrm{t})$, into an orthonormal basis of wavelets is conceptually similar to the Fourier Transform. The latter however is localised in frequency (or time-scale) only while the former, being localised in frequency and time, allows the local decomposition of Non-stationary time series; a compact, two dimentional, representation may be thus obtained (see Morlet et al., 1982; Torrence and Compo, 1998). The wavelets forming the basis are derived from an integrable zero-mean mother wavelet $\psi(\mathrm{t})$ and the wavelet transform of $\mathrm{F}(\mathrm{t})$, be it $\mathrm{W}(\mathrm{t}, \mathrm{f})$, is caculated as the convolution of this function with the mother wavelet duly shifted and scaled in time $\psi(f \cdot(\tau-t))$ :

$$
\mathrm{W}(\mathrm{t}, \mathrm{f})=\int_{-\infty}^{+\infty} \mathrm{F}(\tau) \sqrt{\mathrm{f}} \psi^{*}(\mathrm{f}(\tau-\mathrm{t})) \mathrm{d} \tau
$$

where ${ }^{*}$ denotes complex conjugate, the scale factor $f$ represents frequency and $\sqrt{f}$ is necessary to satisfy the normalization condition; the wavelet transform represents a mapping of $\mathrm{F}(\mathrm{t})$ on the $\mathrm{t}$-f plane.

The mother wavelet which in our case is the Morlet wavelet which consists of a plane wave modulated by a Gaussian: $\psi_{\mathrm{n}}(\mathrm{f})=\pi^{1 / 4} \exp (\mathrm{i} \omega \mathrm{n}) \cdot \exp \left(-\mathrm{n}^{2} / 2\right)$; and $\omega$ is a constant (usually set to 6 , see Torrence and Compo, 1998). This type of mother wavelet is quite common in astrophysical signals analysis facilitating comparison with previously published works. Due to its Gaussian support, the Morlet wavelet expansion inherits optimality as regards the uncertainty principle (Morlet et al., 1982).

The average of the wavelet power spectral density $\|\mathrm{W}(\mathrm{t}, \mathrm{f})\|^{2}$ on time (t) is the global wavelet spectrum (see Torrence and Compo, 1998) and is given by:

$$
\overline{\mathrm{W}(\mathrm{f})}=\frac{1}{\mathrm{~N}} \sum_{\mathrm{n}=1}^{N}\left\|\mathrm{~W}_{\mathrm{n}}(\mathrm{f})\right\|^{2}
$$

for discrete time. The global wavelet spectrum is an unbiased and consistent estimation of the true power spectrum of a time series and generally exhibits similar features and shape as the corresponding Fourier spectrum. 

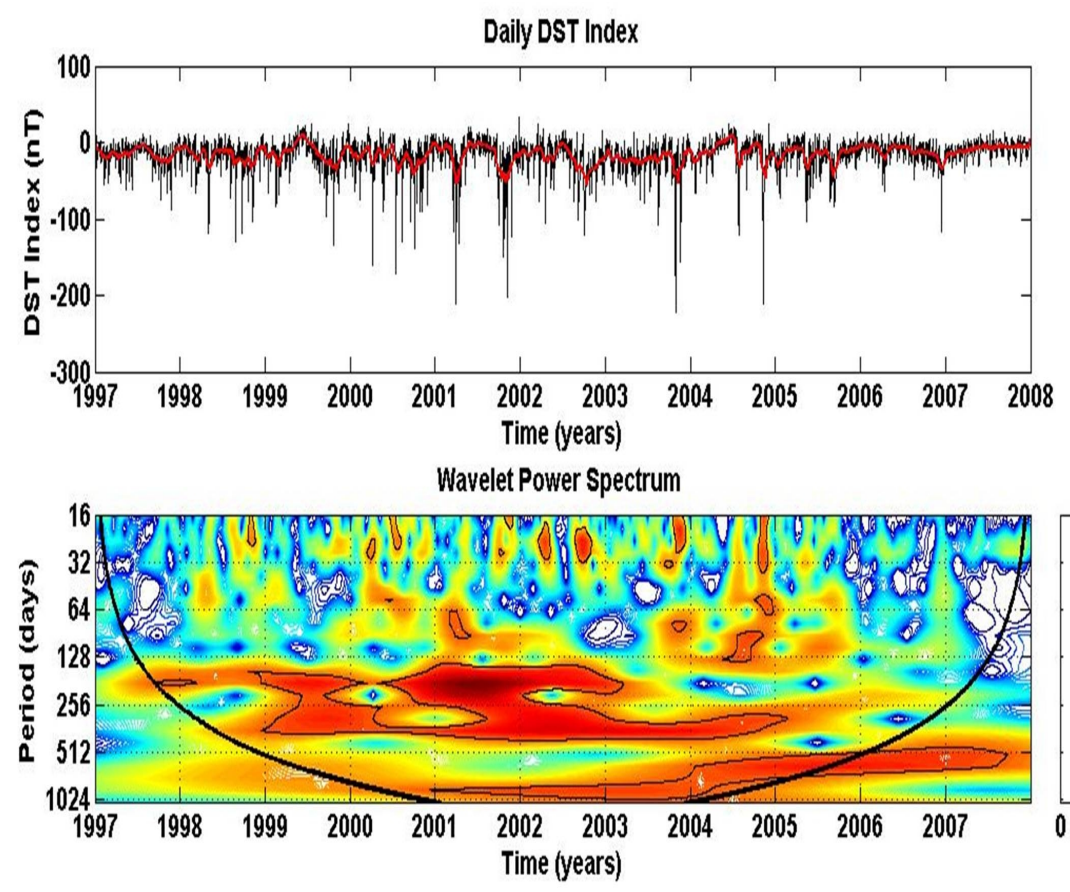

Global Wavelet Spectrum

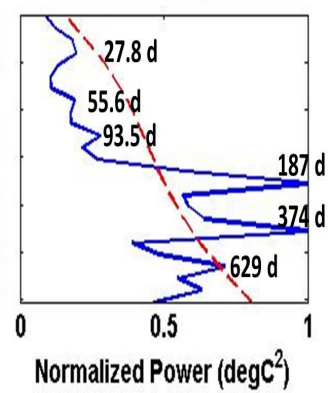

Daily AE Index
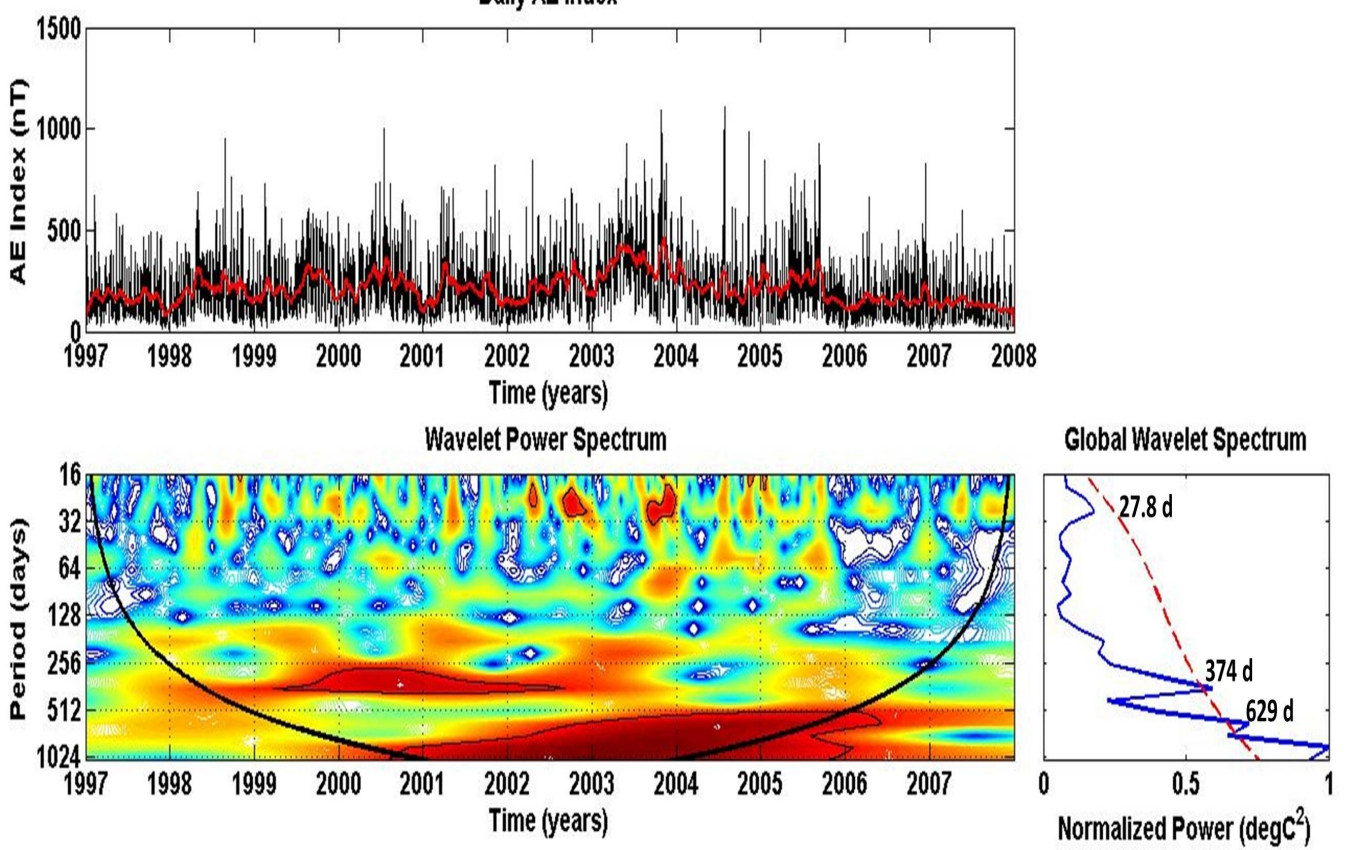

Figure 3: Same with figures 1 and 2 but for geomagnetic indices (top to bottom): $\mathrm{D}_{\mathrm{ST}}$ and AE. 


\subsection{Periodicities in Geomagnetic Indices and Solar Wind Drivers}

From the wavelet power spectra and the global wavelet spectra, presented in 3.1, we identified periodic components of the time-series in 2, in the range from days to a year, within a confidence leve ${ }^{4}$ of $95 \%$. We should note, at this point, that due to the statistical nature of the methodology in use, and the dependence of the results on sample size, we have retained some global peaks that are a little below the $95 \%$ threshold but persist for long periods of time in the power spectrum. The periodicities in the time series appear to dominate certain time intervals being absent from others.

In figures 1 , 3 we identify short (close to the solar rotation) and mid-term (more than 3 months) periodicities of varying power, localized in time. In each of these figures we present the time-series (top panel) to be analysed, the wavelet power spectrum which depicts the time localized periodicities (bottom panel) and the global wavelet spectrum (bottom right panel); the latter is the average over time of each periodic component and facilitates the identification of the peak of each range of periodicities. The studied time-series are described below:

- ICME rate (Figure 1): The mid-term CME periodicities at 187 and 374 days (approximately six and twelve months) at the solar cycle 23 maximum (Polygiannakis et al., 2002; Lou et al., 2003), are present in the ICME rate time series under the $95 \%$ confidence level; a prominent peak of $\sim 187$ days appears only during 2001. Moreover a peak at 66 days appears during 2001 and late 2003. Sporadic short-term periodicities, peak at approximately 25 days, are also present, around the solar maximum (1999-2002) yet they are mostly below the $95 \%$ confidence level of the global spectrum.

- CIR occurrence rate (Figure 2): Ephemeral periodicities (peak at 27.8 days) are most pronounced during the decline phase of the solar cycle 23 yet generally below the $95 \%$ confidence level. The global wavelet spectrum also shows mid-term periodicities with peaks at $\sim 111$ and $\sim 264$ days which appear during the decline and rising phase respectively but with power levels under the $95 \%$ confidence limit.

\footnotetext{
${ }^{4}$ The confidence level is defined as the probability that the true wavelet power at a certain time and scale lies within a certain interval around the estimated wavelet power.
} 
- Geomagnetic Indices (Figure 3): Both indices time-series have intermittent, short-term, sporadic, low-confidence (mostly less than 95\%) periodic components mostly during the late solar maximum and the decline phase (2002-2004). The $\mathrm{D}_{\mathrm{ST}}$ exhibits a pronounced, 374 days, peak (annual periodicity, confidence level exceeds 95\%) in 1999-2004 and a second, 187 days (semi annual periodicity), peak in 1998-2003. On the other hand, AE exhibits only the annual periodicity in the 1999-2002 interval.

The periodic terms common to two or more time-series were analyzed further in the following subsections, using XWT and WTC.

\subsection{Cross Wavelet Analysis and Wavelet Coherence}

The Cross Wavelet Transform (XWT) makes use of the wavelet analysis in the examination of causal relationships in time frequency space between two time series $\mathrm{X}$ and $\mathrm{Y}$ with corresponding CWTs: $\mathrm{W}_{\mathrm{n}}^{\mathrm{X}}(\mathrm{f})$ and $\mathrm{W}_{\mathrm{n}}^{\mathrm{Y}}(\mathrm{f})$. The cross-wavelet transform of the time-series $\mathrm{X}$ and $\mathrm{Y}$ is defined as: $\mathrm{W}_{\mathrm{n}}^{\mathrm{XY}}(\mathrm{f})=\mathrm{W}_{\mathrm{n}}^{\mathrm{X}}(\mathrm{f}) \cdot \mathrm{W}_{\mathrm{n}}^{\mathrm{Y}}(\mathrm{f})^{*}$, with $*$ denoting complex conjugate.

The result is, in general, complex; the modulus, $\left\|\mathrm{W}_{\mathrm{n}}^{\mathrm{XY}}\right\|$, indicates regions in the (t-f) space of high common power and the phase, $\arg \left(\mathrm{W}_{\mathrm{n}}^{\mathrm{XY}}\right)$, of the XWT represents relative phase relationship of the time-series to be compared:

$$
\tan ^{-1}\left[\frac{\operatorname{Im}\left(\left|\mathrm{W}_{\mathrm{n}}^{\mathrm{XY}}(\mathrm{s})\right|\right)}{\operatorname{Re}\left(\left|\mathrm{W}_{\mathrm{n}}^{\mathrm{XY}}(\mathrm{s})\right|\right)}\right]
$$

Said regions of high common power and consistent phase relationship suggest causal relationship between X and Y. From the phase of the XWT a measure of Wavelet Coherence (WTC) between $\mathrm{W}_{\mathrm{n}}^{\mathrm{X}}$ and $\mathrm{W}_{\mathrm{n}}^{\mathrm{Y}}$ will be derived below. The statistical significance of the Cross Wavelet Spectrum was estimated following Torrence and Compo (1998) and Grinsted (2006).

The Cross-Wavelet Transform is used in the calculation of the degree of cause and effect dependence of the geomagnetic response to the Solar Activity and the Solar Wind as all of them are represented by time series.

The Wavelet Coherence (WTC) is an estimator of the confidence level for each detection of a time-space region of high common power and consistent phase relationship, calculated by the Cross Wavelet Transform, between two time-series. The measure of wavelet coherence is defined between two continuous wavelet transforms and it may indicate coherence with high confidence level even though the common power is low; it closely resembles a localized 
correlation coefficient in time-frequency space and varies between 0 and 1 . It is used alongside the Cross Wavelet Transform as the latter appears to be unsuitable for significance testing the interrelation between two processes (Maraun and Kurths, 2004). Following Torrence and Webster (1998) we define the wavelet coherence of two time series, let them be $\mathrm{X}$ and $\mathrm{Y}$ :

$$
\mathrm{R}_{\mathrm{n}}^{2}(\mathrm{f})=\frac{\left|\mathrm{S}\left(\mathrm{f}^{-1} \mathrm{~W}_{\mathrm{n}}^{\mathrm{XY}}(\mathrm{f})\right)\right|^{2}}{\mathrm{~S}\left(\mathrm{f}^{-1}\left|\mathrm{~W}_{\mathrm{n}}^{\mathrm{X}}(\mathrm{f})\right|^{2}\right) \cdot \mathrm{S}\left(\mathrm{f}^{-1}\left|\mathrm{~W}_{\mathrm{n}}^{\mathrm{Y}}(\mathrm{f})\right|^{2}\right)}
$$

where $\mathrm{S}$ is a smoothing operator. As this definition closely resembles that of a traditional correlation coefficient, we might think the wavelet coherence as a correlation coefficient localized in time frequency space. The statistical significance level of the wavelet coherence is estimated using Monte Carlo methods.

A detailed description of the Wavelet-Based Method for the Comparison of Time Series may be found in Torrence and Webster (1998); Grinsted et al. (2004); Grinsted (2006). The Matlab package of the National Oceanography Centre, Liverpool, UK5 was used in the calculation of the WXT and WTC.

\subsection{ICMEs-Geomagnetic Effects Relationship}

Figure 4, shows the cross-wavelet transform and wavelet coherence calculations used to study the interrelation of ICMEs and geomagnetic indices. The middle panels show the cross-wavelet spectrum (XWT, see 3.3) of the two time series under examination; the common power of the time-series pair is colour coded in the time-period domain. The left panels depict the time average of the XWT spectrum and the right panels the wavelet coherence. The latter is the correlation coefficient (Equation 4) of the time-series wavelet transform phase. Arrows indicate the phase relationship between the two data series (Equation 3), in time-frequency space: Those pointing to the right correspond to in-phase behavior those to the left anti-phase. The downwards pointing arrows indicate $90^{\circ}$ lead of the first data-set. Since geomagnetic strorms imply large negative values of $\mathrm{D}_{\mathrm{ST}}$ the convention is reversed and now left indicates in phase and downwards pointing arrows imply that the ICME time series leads the $\mathrm{D}_{\mathrm{ST}}$. The same reversed convention holds in section 3.5 for the CIR- $\mathrm{D}_{\mathrm{ST}}$ time series.

\footnotetext{
${ }^{5}$ http://noc.ac.uk/usingscience/crosswaveletwaveletcoherence
} 

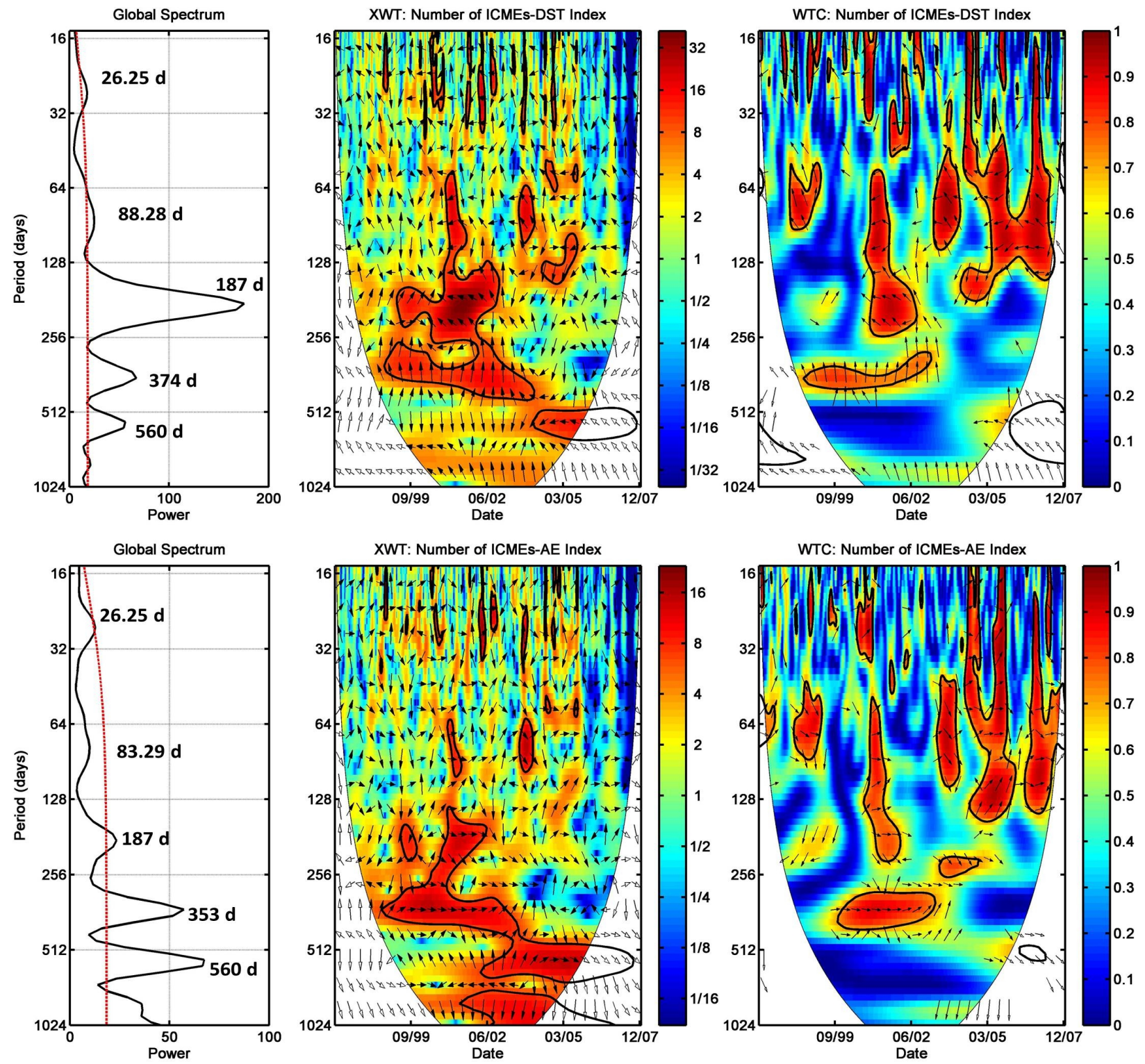

Figure 4: Global wavelet (left), Cross-wavelet transformation (XWT, middle) and Wavelet Coherence (WTC, right) of the ICME occurrence rate and the geomagnetic indices; The dashed red line corresponds to the $95 \%$ confidence level of the global wavelet. The thick black contours mark the $95 \%$ confidence level, and the the thin line indicates the cone of influence (COI). The colour-bar of the XWT indicates the power of period range; the colour-bar of the WTC corresponds to the significance level of the Monte-Carlo test. The arrows point to the phase relationship of the two data series in time-frequency space: (1) arrows pointing to the right show in-phase behavior; (2) arrows pointing to the left indicate anti-phase behavior; (3) arrows pointing downward indicate that the first dataset is leading the second by $90^{\circ}$ 
Similar to Katsavrias et al. (2012), we consider significant, in XWT, WTC and in continuous wavelet, the shared periodicities which persist for an interval of at least $4-5$ times its period and with a coherence coefficient above 0.8 .

We discuss below the approximately 27 days, 3 month, semi-annual, annual and 560 days periodicity. Those represent the pronounced peaks of the geomagnetic disturbances and their drivers, cross wavelet transform (XWT):

- ICMEs-D $\mathrm{D}_{\mathrm{ST}}$ index: The ICME rate and the $\mathrm{D}_{\mathrm{ST}}$ index share the annual periodicity (peak at the 374 days) most of the solar cycle 23 (19982003) with generally phase-locked behavior; the ICME rate leads by $90^{\circ}$ the $\mathrm{D}_{\mathrm{ST}}$. The first harmonic (187 days peak), appears in 1999-2002 with varying phase behavior. Short-term 26 days periodicity appears intermittently in short intervals throughout the cycle; prominent peaks appear 1999, 2001 and 2005 with varying phase ICME-- $\mathrm{ST}_{\mathrm{ST}}$ behaviour. A prominent peak at approximately 88 days is also present at 2001 and late 2003.

- ICMEs-AE index: The ICME rate and the AE index time-series share the, approximately, annual and semi-annual periodicities (peaks at 353 and 187 days) in 1999-2002 yet only the former exhibits in-phase relationship. Short-term, 27 days, periodicities appear sporadically throughout the cycle 23 with varying phase behavior. The approximately 3 months periodicity (peak at 83 days) is also present but mostly below the $95 \%$ confidence level.

The 560 days periodicity appears in both ICME-D $\mathrm{D}_{\mathrm{ST}}$ and ICME-AE XWT during the whole solar cycle yet is above the $95 \%$ confidence level only during 2004-2005 and 2002-2005 time intervals respectively.

\subsection{CIR-Geomagnetic Effects Relationship}

We examine the CIR rate relationship to the $\mathrm{D}_{\mathrm{ST}}$ and $\mathrm{AE}$ time series. The results of the cross-wavelet transform (XWT) and wavelet coherence (WTC) are presented in figure 5, in the same form as in section 3.4, and described below:

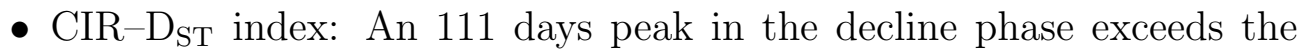
$95 \%$ confidence level in 2005. There is an approximately semi-annual, 198 days peak that exceeds the $95 \%$ confidence level in 2001-2002 but is 

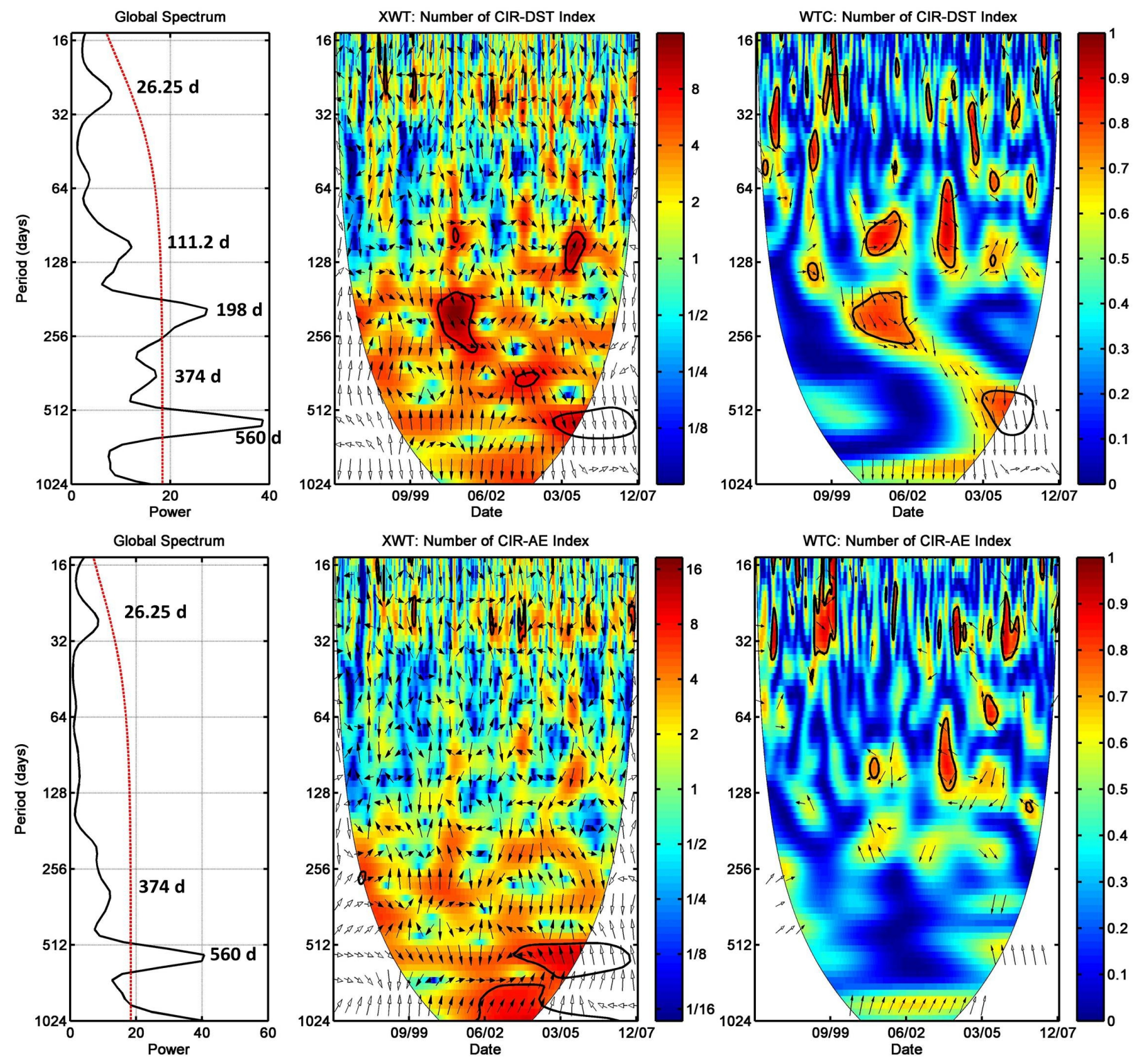

Figure 5: Cross-wavelet transformation (left) and Wavelet Coherence (right) of the CIR occurrence and the geomagnetic indices, same as Figure 4 

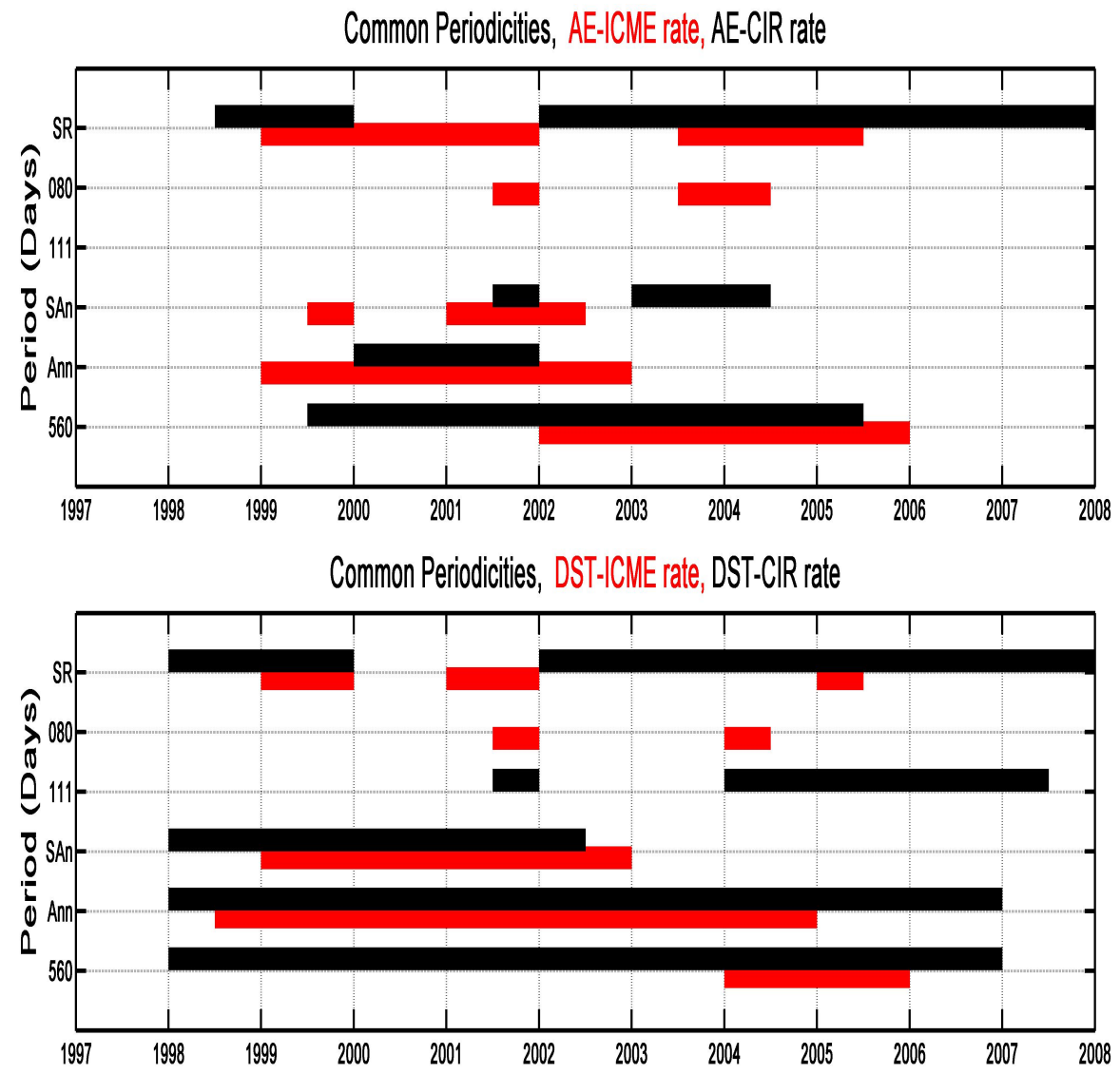

Figure 6: Common periodicities between geomagnetic indices (AE in upper panel and $\mathrm{D}_{\mathrm{ST}}$ in the lower panel) and drivers as detected by the XWT. The red lines correspond to ICMEs and the black to CIR. The y-axis labels SR, SAn and Ann stand for Solar Rotation, Semi-Annual and Annual periodicities. 
also present in the rising and maximum phase of the cycle. The annual periodicity is present, with very low confidence level, in the entire cycle. In all cases the phase relationship is varying.

- CIR-AE index: The cross wavelet spectrum (XWT) of the CIR rateAE index time series has a low confidence (less than 95\%) peak (374 days) during the maximum of the cycle (semi-annual periodicity was not detected).

- Intermittent, and, mostly low confidence (under 95\%) 27 days (ephemeral) peaks appear during the rising and decline phase of the cycle. This behavior is common to the ICME rate the $\mathrm{D}_{\mathrm{ST}}$ and the AE, time series.

The 560 days periodicity appears in both CIR-D ST $_{\text {and CIR-AE XWT during }}$ the whole solar cycle yet is above the $95 \%$ confidence level only during the decline phase.

\subsection{Discussion}

In this report two geomagnetic indices (daily values), each quantifying a different magnetospheric process ( $\mathrm{D}_{\mathrm{ST}}$ for ring current and $\mathrm{AE}$ for substorms) were examined. In place of the transient phenomena we used the daily number of ICMEs occurrence and for the recurrent phenomena the daily number of CIRs occurrence; these are the two drivers of the separate components.

The results in subsection 3.1 reveal short to medium periodicities in the range of days up to the year within the solar cycle 23. They are consistent with previous work by Katsavrias et al. (2012) where similar periodicities were detected within a sample spanning four solar cycles. In their report a number of spectral peaks where found with confidence exceeding 99\%. In this study, due to the smaller sample, most of these peaks appear below the $95 \%$ limit.

The ICME rate time-series has three significant components with periods of about 25, 66 and 187 days respectively; they are both quite pronounced during the rising phase and maximum of the cycle (see Fig. 1). This result is, in part, consistent with the $\approx 100-200$ days periodicities (including the 153 day periodicity by Rieger et al., 1984) of ICMEs per solar rotation reported by Richardson and Cane (2005) and Richardson and Cane (2010), during the maximum and the decline phase (2004-2005). On the other hand, the CIR rate has two pronounced frequency components of about 27 and 
111 days in the decline phase of the cycle (see Fig. 2). The wavelet spectra of the geomagnetic indices $\mathrm{D}_{\mathrm{ST}}$ and $\mathrm{AE}$ show also the 27 days periodicity and, in addition, a strong annual component (peak at 374 days) while the semi-annual variation appears pronounced only in the $\mathrm{D}_{\mathrm{ST}}$ index. The latter is probably the result of the Russell and McPherron (1973) effect which links the Earth's orbital position to the southward component of the interplanetary magnetic field; the ring current (and $\mathrm{D}_{\mathrm{ST}}$ ), being associated to the dayside-reconnection which depends strongly on this component, is significantly affected. This is not the case with the AE index as this is driven, mostly, by ram-pressure at the magnetotail (night-side reconnection) and is not as sensitive to the southward component.

Quite often the effects of these drivers are not distinct so the drivercomponent pairs are not easily separable in time, yet, by means of wavelet analysis (CWT, XWT, WTC) separation in the time-frequency plain may be obtained. Our examination indicates certain periods in time, or intervals in frequency (period) where some component becomes dominant for one or more indices.

The unusually active decline phase of solar cycle 23 (see Kossobokov et al., 2012, and references within) is an example of the importance of such time-frequency separability because, although the fast solar wind geomagnetic effects are dominant, there is also a significant contribution from transient flows (ICMEs) which originated from a higher-than-expected number of CMEs. Under normal circumstances the CIR-driven storms should generally occur in the the rise phase and then into the late declining phase of the solar cycle while the CME-driven storm should prevail at solar maximum (Gonzalez et al., 1999; Yermolaev and Yermolaev, 2002) as CME occurrence frequency and their velocity are both greatest during solar maximum (Gopalswamy et al., 2004). The separation between the two is, within the decline phase, only possible in the frequency (period) space as the shared shortterm periodicities between ICMEs and geomagnetic indices and the shared short-term periodicities between CIRs and geomagnetic indices, both quite pronounced, do not overlap.

The examination of the drivers-Geomagnetic Effects relationship, by means of cross wavelet spectra and wavelet coherence, in subsection 3.4 and 3.5 are summarized in figure6. The length of each bar in the chart represents the interval where common periodicities between driver and magnetospheric index are pronounced in the cross-power spectra (WXT). It indicates the following:

In the In the 27 day periodicities the CIRs modulate the two geomagnetic 
indices with interference from ICMEs during the cycle maximum and the extremely active period 2003; the 27 days CIR modulation of the geomagnetic indices does not appear at the cycle maximum where the major driver is the ICMEs. As regards annual and semi-annual periodicities (374 and 187 days) the ICME and CIR modulation of the geomagnetic disturbances overlap throughout the cycle 23. The 560 days periodicity, on the other hand, is dominated by CIRs during the whole cycle; the ICME contribution is localized within the unusually active decline phase 2002-2005(see Fig. 6).

The results, presented above, point to an anti-correlation of recurrent and transient phenomena in both geomagnetic indices as regards the 27 days component (see Fig. 6). This is consistent, in part, with the results of Feynman (1982) and $\mathrm{Du}$ (2011) which demonstrated the anti-correlation of the recurrent and transient effects for a number of solar cycles using, however, low resolution data. For the component with period of 1.5 year the CIR seem to be the dominant driver again for both indices. For the remaining components, presented also in figure 6, the driver-responce relationship is more complex and is not always the same for the $\mathrm{D}_{\mathrm{ST}}$ and $\mathrm{AE}$.

The common periodicities shared between ICMEs and the geomagnetic indices show prolonged periods of phase-locked behaviour (i.e consistent phase relationship) for components with periods of about a year. Furthermore, the ICME rate leads DST by $90^{\circ}$ which corresponds to a time lag of about 3 months whereas, in contrast, the ICME rate is in phase with AE index. The physical origin of this intriguing difference between the phasing of Dst and AE relative to the ICME rate requires further investigation and is beyond the scope of this paper.

The common periodicities shared between CIRs-Geomagnetic indices show strong variations concerning the phase relationship as the CIR associated magnetic field, and the highly geoeffective z-component in particular, fluctuates strongly in a complex way.

\section{Conclusions}

In the present study the relationship between transient (sporadic) and recurrent phenomena, ICMEs and CIRs, and the corresponding magnetospheric response represented by geomagnetic indices $\left(\mathrm{D}_{\mathrm{ST}}\right.$ and $\left.\mathrm{AE}\right)$ was examined. For the examination of this relationship between the drivers and the corresponding magnetospheric response we used the cross-wavelet transform (XWT), and wavelet coherence (WTC). 
Our results indicate that:

1. CIRs modulate the geomagnetic responce during the rise and decline phase while ICMEs during the maximum of the cycle and the unusual active period of 2002-2005; the phase-relationship varies strongly in all cases for both drivers. Therefore there is an anti-correlation of recurrent and transient/sporadic phenomena throughout the solar cycle 23 but it is evident in the 27-days periodic component only.

2. The only clear phase-locked behaviour was found in the XWT of the ICME-D ${ }_{\mathrm{ST}}$, ICME-AE components with periods of $\approx 1.0$ year. In the ICME- $D_{\mathrm{ST}}$ case the phase difference corresponded to a time lag of about three months, while the ICME-AE XWT exhibited in-phase behaviour.

3 . The component with period of $\approx 1.3-1.7$ years of the CIR time-series seem to be the dominant driver for both indices throughout the whole solar cycle 23 .

A future study with a larger data-set (exceeding one solar cycle) is necessary in order to verify these results and expand in larger time-scales.

\section{Acknowledgement}

The free for non-profit use MATLAB package of the National Oceanography Centre, Liverpool, UK was used in this work. The authors thank S. Patsourakos and the anomymous referees for helpful suggestions.

\section{References}

Akasofu, S.-I., Dec. 2011. A Historical Review of the Geomagnetic StormProducing Plasma Flows from the Sun. Space Sci. Rev. 164, 85-132.

Borovsky, J. E., Denton, M. H., Jul. 2006. Differences between CME-driven storms and CIR-driven storms. J. Geophys. Res. 111, A07S08.

Cliver, E. W., 1995. Solar activity and geomagnetic storms: From M regions and flares to coronal holes and CMEs. EOS Transactions 76, 75-75.

Clúa de Gonzalez, A. L., Gonzalez, W. D., Dutra, S. L. G., Tsurutani, B. T., Jun. 1993. Periodic variation in the geomagnetic activity: A study based on the Ap index. J. Geophys. Res. 98, 9215-9232. 
Deng, L. H., Li, B., Zheng, Y. F., Cheng, X. M., Oct. 2013. Relative phase analyses of $10.7 \mathrm{~cm}$ solar radio flux with sunspot numbers. New Astronomy $23,1-5$.

Deng, L. H., Qu, Z. Q., Wang, K. R., Li, X. B., Nov. 2012. Phase asynchrony between coronal index and sunspot numbers. Adv. Space Res. 50, 14251433.

Du, Z. L., Aug. 2011. The correlation between solar and geomagnetic activity - Part 1: Two-term decomposition of geomagnetic activity. Ann. Geophys. 29, 1331-1340.

Fenimore, E. E., Asbridge, J. R., Bame, S. J., Feldman, W. C., Gosling, J. T., Sep. 1978. The power spectrum of the solar wind speed for periods greater than 10 days. J. Geophys. Res. 83, 4353-4357.

Feynman, J., Aug. 1982. Geomagnetic and solar wind cycles, 1900-1975. J. Geophys. Res. 87, 6153-6162.

Gonzalez, A. L. C., Gonzalez, W. D., May 1987. Periodicities in the interplanetary magnetic field polarity. J. Geophys. Res. 92, 4357-4375.

Gonzalez, W. D., Tsurutani, B. T., Clúa de Gonzalez, A. L., Apr. 1999. Interplanetary origin of geomagnetic storms. Space Sci. Rev. 88, 529-562.

Gopalswamy, N., Nunes, S., Yashiro, S., Howard, R. A., Jan. 2004. Variability of solar eruptions during cycle 23. Adv. Space Res. 34, 391-396.

Grinsted, A., 2006. Advanced methods of glaciological modelling and time series analysis. Københavns Universitet', The Niels Bohr Institute, Ice and Climate.

Grinsted, A., Moore, J. C., Jevrejeva, S., Nov. 2004. Application of the cross wavelet transform and wavelet coherence to geophysical time series. Nonlinear Processes in Geophysics 11, 561-566.

Jian, L., Russell, C. T., Luhmann, J. G., Skoug, R. M., Dec. 2006a. Properties of Interplanetary Coronal Mass Ejections at One AU During 1995-2004. Solar Phys. 239, 393-436. 
Jian, L., Russell, C. T., Luhmann, J. G., Skoug, R. M., Dec. 2006b. Properties of Stream Interactions at One AU During 1995 2004. Solar Phys. 239, 337-392.

Katsavrias, C., Preka-Papadema, P., Moussas, X., Oct. 2012. Wavelet Analysis on Solar Wind Parameters and Geomagnetic Indices. Solar Phys. 280, 623-640.

Kossobokov, V., Le Mouël, J.-L., Courtillot, V., Feb. 2012. On Solar Flares and Cycle 23. Solar Phys. 276, 383-394.

Kudela, K., Mavromichalaki, H., Papaioannou, A., Gerontidou, M., Sep. 2010. On Mid-Term Periodicities in Cosmic Rays. Solar Phys. 266, 173180.

Lou, Y.-Q., Wang, Y.-M., Fan, Z., Wang, S., Wang, J. X., Nov. 2003. Periodicities in solar coronal mass ejections. Mon. Not. Roy. Astron. Soc. 345, 809-818.

Maraun, D., Kurths, J., Nov. 2004. Cross wavelet analysis: significance testing and pitfalls. Nonlinear Processes in Geophysics 11, 505-514.

Mavromichalaki, H., Preka-Papadema, P., Petropoulos, B., Tsagouri, I., Georgakopoulos, S., Polygiannakis, J., Aug. 2003. Low- and high-frequency spectral behavior of cosmic-ray intensity for the period 1953-1996. Annales Geophysicae 21, 1681-1689.

Mayaud, P. N., 1980. Derivation, Meaning, and Use of Geomagnetic Indices. Washington DC American Geophysical Union Geophysical Monograph Series 22,607 .

Mitsakou, E., Moussas, X., Aug. 2014. Statistical Study of ICMEs and Their Sheaths During Solar Cycle 23 (1996 - 2008). Solar Phys. 289, 3137-3157.

Morlet, J., Arens, G., Forgeau, I., Giard, D., 1982. Wave Propagation and Sampling Theory. Geophysics 47, 203-236.

Mursula, K., 1999. Simultaneous Occurrence of Mid-term Periodicities in Solar Wind Speed, Geomagnetic Activity and Cosmic Rays. In: D. Kieda, M. Salamon, B. Dingus (Ed.), IUPAP. Proceedings of the 26th International Cosmic Ray Conference. Vol. 7. p. 123. 
Polygiannakis, J., Preka-Papadema, P., Petropoulos, B., Pothitakis, G., Moussas, X., Pappas, G., Hillaris, A., Oct. 2002. Ephemeral periodicities in the solar activity. In: H. Sawaya-Lacoste (Ed.), SOLMAG 2002. Proceedings of the Magnetic Coupling of the Solar Atmosphere Euroconference. Vol. 505 of ESA-SP. pp. 537-540.

Prabhakaran Nayar, S. R., Radhika, V. N., Revathy, K., Ramadas, V., Aug. 2002. Wavelet Analysis of solar, solar wind and geomagnetic parameters. Solar Phys. 208, 359-373.

Pulkkinen, T., May 2007. Space Weather: Terrestrial Perspective. Living Reviews in Solar Physics 4, 1.

Richardson, I. G., Cane, H. V., Jan. 2005. The 150 day quasi-periodicity in interplanetary and solar phenomena during cycle 23. Geophys. Res. Lett. 32, 2104.

Richardson, I. G., Cane, H. V., Jun. 2010. Near-Earth Interplanetary Coronal Mass Ejections During Solar Cycle 23 (1996-2009): Catalog and Summary of Properties. Solar Phys. 264, 189-237.

Richardson, I. G., Cane, H. V., May 2012. Solar wind drivers of geomagnetic storms during more than four solar cycles. Journal of Space Weather and Space Climate 2 (26), A260000.

Rieger, E., Kanbach, G., Reppin, C., Share, G. H., Forrest, D. J., Chupp, E. L., Dec. 1984. A 154-day periodicity in the occurrence of hard solar flares? Nature 312, 623-625.

Russell, C. T., McPherron, R. L., 1973. Semiannual variation of geomagnetic activity. J. Geophys. Res. 78 (1), 92-108.

Sabbah, I., Kudela, K., Apr. 2011. Third harmonic of the 27 day periodicity of galactic cosmic rays: Coupling with interplanetary parameters. $J$. Geophys. Res. 116, 4103.

Schwenn, R., Aug. 2006. Space Weather: The Solar Perspective. Living Reviews in Solar Physics 3, 2.

Svalgaard, L., Wilcox, J. M., Apr. 1975. Long-term evolution of solar sector structure. Solar Phys. 41, 461-475. 
Torrence, C., Compo, G. P., Jan. 1998. A Practical Guide to Wavelet Analysis. Bull. Amer. Meteor. Soc. 79, 61-78.

Torrence, C., Webster, P. J., Jul. 1998. The annual cycle of persistence in the El Nño/Southern Oscillation. Quarterly Journal of the Royal Meteorological Society 124, 1985-2004.

Valdés-Galicia, J. F., Pérez-Enríquez, R., Otaola, J. A., Aug. 1996. The Cosmic-Ray 1.68-Year Variation: a Clue to Understand the Nature of the Solar Cycle? Solar Phys. 167, 409-417.

Valdés-Galicia, J. F., Velasco, V. M., 2008. Variations of mid-term periodicities in solar activity physical phenomena. Adv. Space Res. 41, 297-305.

Yermolaev, Y. I., Yermolaev, M. Y., Jan. 2002. Statistical Relationships between Solar, Interplanetary, and Geomagnetospheric Disturbances, 19762000. Cosmic Research 40, 1-14. 